\title{
Output power PDF of a saturated semiconductor optical amplifier: Second-order noise contributions by path integral method
}

\author{
Öhman, Filip; Mørk, Jesper; Tromborg, Bjarne
}

Published in:

I E E E Journal of Quantum Electronics

Link to article, DOI:

10.1109/JQE.2007.906226

Publication date:

2007

Document Version

Publisher's PDF, also known as Version of record

Link back to DTU Orbit

Citation (APA):

Öhman, F., Mørk, J., \& Tromborg, B. (2007). Output power PDF of a saturated semiconductor optical amplifier: Second-order noise contributions by path integral method. I E E E Journal of Quantum Electronics, 43(12), 1188-1197. https://doi.org/10.1109/JQE.2007.906226

\section{General rights}

Copyright and moral rights for the publications made accessible in the public portal are retained by the authors and/or other copyright owners and it is a condition of accessing publications that users recognise and abide by the legal requirements associated with these rights.

- Users may download and print one copy of any publication from the public portal for the purpose of private study or research.

- You may not further distribute the material or use it for any profit-making activity or commercial gain

- You may freely distribute the URL identifying the publication in the public portal 


\title{
Output Power PDF of a Saturated Semiconductor Optical Amplifier: Second-Order Noise Contributions by Path Integral Method
}

\author{
Filip Öhman, Jesper Mørk, and Bjarne Tromborg
}

\begin{abstract}
We have developed a second-order small-signal model for describing the nonlinear redistribution of noise in a saturated semiconductor optical amplifier. In this paper, the details of the model are presented. A numerical example is used to compare the model to statistical simulations. We show that the proper inclusion of second-order noise terms is required for describing the change in the skewness (third-order moment) of the noise distributions. The calculated probability density functions are described far out in the tails and can hence describe signals with very low bit error rate (BER). The work is relevant for describing the noise distribution and BER in, for example, optical regeneration.
\end{abstract}

Index Terms-Noise, optical communication, optical signal processing, semiconductor optical amplifiers (SOAs).

\section{INTRODUCTION}

$\mathbf{S}$ EMICONDUCTOR optical amplifiers (SOAs) have a number of promising applications within optical communication systems. Some examples are all-optical wavelength conversion [1], [2], regeneration [3], [4], limiting amplification [5], and noise suppression in spectrum-sliced wavelength-division multiplexed systems [6]. In these applications, the performance, as measured by the bit error rate (BER), depends on the noise distribution of the signal after the SOAs. It is therefore important to describe the noise in an SOA in detail. However, the saturation and nonlinear properties of the SOA make this description complicated. Shtaif and coworkers used a first-order perturbation analysis for examining the noise spectra after a saturated SOA [7] and experimentally measured the noise distribution [8]. Bilenca and colleagues have made a detailed study of noise distributions in SOAs using multicanonical Monte Carlo simulation [9] and Fokker-Planck equations [10]. We have previously measured and calculated the probability density functions (PDFs) after amplification in a saturated SOA [11]. The calculations of the PDFs were based on statistical simulations, standard assumptions like Gaussian or noncentral $\chi^{2}$-distributions, or used models [12] that are not able to describe the nonlinear noise redistribution shown in experiments [11], [13]. Large-signal simulations can, in principle, include the nonlinear redistribution, but it is difficult

Manuscript received January 18, 2007; revised June 27, 2007. This work was supported in part by the IST Project BIGBAND, the European Network of Excellence ePIXnet, and the Danish Technical Research Council.

The authors are with COM•DTU Institute for Communication, Optics and Materials, NanoDTU, Technical University of Denmark, DK-2800 Kgs. Lyngby, Denmark (e-mail: fo@com.dtu.dk; jm@com.dtu.dk; btromborg@mail.dk).

Digital Object Identifier 10.1109/JQE.2007.906226 to reach far out in the tails of the distributions, i.e., to simulate the rare occurrences of errors corresponding to low BERs. One way of expanding the range of BERs by simulations is through importance sampling [9], but efficient application of that method is still somewhat of an art [14], [15]. In this study, we have developed a detailed analytical model that, in principle, allows calculation of the PDF of a noisy signal at the output of an SOA including the tails of very low probability densities. The model is an extension of the standard noncentral $\chi^{2}$-distribution, which takes into account additional second-order noise contributions in the sense discussed in the following.

We examine the noise properties of a generic type of SOA. The optical amplification in the SOA is assumed to take place in a waveguide of length $L$, and the electrical field in the waveguide is described by its complex envelope $E(z, t)$ normalized such that $|E|^{2}$ is the optical power. By solving the equations for the propagation of the electrical field through the SOA, one can determine the output field $E(L, t)$ in terms of the input signal field $E(0, t)$ and the spontaneous emission noise. The spontaneous emission is added and amplified during transmission, and it interacts in a nonlinear manner with the signal. In this paper, we shall only consider the case where $E(0, t)$ is a $C W$ signal to which we have added Gaussian noise terms. The field $E(z, t)$ can then be expanded as

$$
\begin{aligned}
E & =\sqrt{P_{\text {sat }}}\left(\rho_{s}+\rho_{1}+\rho_{2}+\cdots\right) e^{i\left(\phi_{s}+\phi_{1}+\phi_{2}+\cdots\right)} \\
& =E_{s}\left(1+\frac{\rho_{1}}{\rho_{s}}+\frac{\rho_{2}}{\rho_{s}}+\cdots\right) e^{i\left(\phi_{1}+\phi_{2}+\cdots\right)}
\end{aligned}
$$

where

$$
E_{s}(z)=\sqrt{P_{\text {sat }}} \rho_{s}(z) e^{i \phi_{s}(z)}
$$

is the steady-state field in the absence of noise, $P_{\text {sat }}$ is the saturation power to be introduced later, and $\phi_{i}(z, t)$ and $\rho_{i}(z, t)$ are the noise contributions to the phase and normalized amplitude of $i$ th order for $i \geq 1$.

The optical output power $P(t)$ is equal to $|E(L, t)|^{2}$, i.e.,

$$
P(t)=P_{s}\left(\left(1+\frac{\rho_{1}}{\rho_{s}}\right)^{2}+2 \frac{\rho_{2}}{\rho_{s}}+\cdots\right)_{z=L}
$$

where $P_{s}=P_{\text {sat }} \rho_{s}^{2}(L)$ is the steady-state power in the absence of noise. In the calculations of the PDF for $P(t)$, the approximation $P(t) \simeq P_{s}\left(1+2 \rho_{1} / \rho_{s}\right)$ gives a Gaussian distribution of $P(t)$, while the approximation $P(t) \simeq P_{s}\left(1+\rho_{1} / \rho_{s}\right)^{2}$ leads to a noncentral $\chi^{2}$-distribution for $P(t)$. The latter includes the second-order term $P_{s}\left(\rho_{1} / \rho_{s}\right)^{2}$, but it does not include the $\rho_{2}$-term, i.e., it does not comprise the full second-order 
noise contribution. The $\rho_{2}$-term contains, among other effects, the gain saturation caused by the copropagating amplified spontaneous emission (ASE). The main results of this paper are the derivation of an analytical expression for the PDF of $P(t)$ to second order and the presentation of approximate methods for calculating the PDF. We also present examples that compare the distribution with the results obtained from a direct large-signal simulation of the time-domain equations for the field and the carrier density in the SOA. The distributions agree over the range in which we can obtain the simulated PDF within a reasonable computation time. Furthermore, we demonstrate that the full second-order calculation may give a BER, which at the level of $10^{-10}$ deviates by an order of magnitude from a noncentral $\chi^{2}$ calculation.

Our present analysis assumes a CW incoming signal, but, in order to deal with most SOA applications (i.e., amplification of digital signals, wavelength conversion, and regeneration), it must be extended to include modulated signals. This can be done following our approach, but the calculations become substantially more numerically demanding. Our analysis also assumes copropagation of signal and ASE. In order to take into account counterpropagating ASE or reflections from the SOA facets, the model must include an extra field equation for the backward propagating field as in laser modeling. It is beyond the scope of this paper to extend the formalism to cope with modulated signals, counterpropagating ASE, or cases of low signal power, where the noise cannot be considered as a perturbation. However, we show that second-order effects become important when calculating low BERs, and we expect this conclusion to persist in analyses that go beyond our simplifying assumptions.

The paper is structured as follows. In Section II, the basic model is presented, while the details of the PDF calculations are presented in Section III and the numerical examples are analyzed and discussed in Section IV. The final conclusions are drawn in Section V. Some of the more detailed derivations are collected in the three Appendices.

\section{NOISE MODEL FOR THE SOA}

The analysis of noise in the SOA is performed in two different ways: a perturbation analysis to second order in the noise contributions and a large-signal simulation. Both approaches are based on a model for the SOA that is described in this section.

The model is a standard rate equation for the carrier density in an SOA and a propagation equation for the electric field $E(z, t)$, as described in [16]. The noise is incorporated in the equations by Langevin forces, in accordance with [7] and [17]. The resulting equations for carrier density and electric field are

$$
\begin{aligned}
& \frac{\partial N}{\partial t}=\frac{I}{q V}-\frac{N}{\tau_{s}}-\frac{g(N)}{A \hbar \omega_{0}}|E|^{2}+f_{N} \\
& \frac{\partial E}{\partial z}=\frac{1}{2}\left(g(1-i \alpha)-\alpha_{\text {int }}\right) E+f_{E}
\end{aligned}
$$

where $I$ is the injected current, $q$ is the elementary charge, $V$ is the active volume, $\tau_{s}$ is the spontaneous carrier lifetime, $A$ is the effective cross-section area of the active region, $\hbar \omega_{0}$ is the photon energy, $\alpha$ is the linewidth enhancement factor, and $\alpha_{\text {int }}$ is the waveguide loss. The time variable $t$ is a shifted time coordinate, $t=t_{\text {real }}-z / v_{\mathrm{g}}$, where $t_{\text {real }}$ is the real time coordinate and $v_{\mathrm{g}}$ is the group velocity. The propagation is unidirectional and perfect anti-reflection coatings are assumed, i.e., the reflectivities of the facets are zero. The gain $g(N)$ is approximated as a linear function of the carrier density, and it is assumed that the carrier frequency $\omega_{0}$ is chosen at the gain peak. The gain is then

$$
g(N)=a\left(N-N_{0}\right)
$$

where $a$ is the differential modal gain and $N_{0}$ is the carrier density at transparency.

The functions $f_{E}$ and $f_{N}$ are Langevin noise terms, where $f_{E}$ describes the spontaneous emission noise and $f_{N}$ describes the carrier density noise imposed by carrier injection and recombination noise. The work in [7] presents a detailed analysis of the influence of carrier density noise on the relative intensity noise (RIN) spectrum of the output signal of a saturated SOA. The analysis shows that, for input powers of about $10 \%$ of the saturation power, the carrier noise only gives a small contribution to the RIN at low frequencies compared with the carrier bandwidth. At higher input powers, the carrier noise becomes increasingly important and may even lead to quantum optical squeezing effects for input powers above the saturation power [18]. In this paper, we only consider cases of moderate saturation, and we shall therefore assume that $f_{N}=0$.

The Langevin function $f_{E}$ for spontaneous emission is considered as a Gaussian noise source with correlation relations

$$
\begin{aligned}
& \left\langle f_{E}\left(z_{1}, t_{1}\right) f_{E}^{*}\left(z_{2}, t_{2}\right)\right\rangle=R_{\mathrm{sp}}\left(z_{1}, t_{1}-t_{2}\right) \delta\left(z_{1}-z_{2}\right) \\
& \left\langle f_{E}\left(z_{1}, t_{1}\right) f_{E}\left(z_{2}, t_{2}\right)\right\rangle=0 .
\end{aligned}
$$

The Fourier transform of $R_{\mathrm{sp}}(z, \tau)$ is the local spontaneous emission spectrum

$$
\begin{aligned}
S_{\mathrm{sp}}(z, \omega) & =\int R_{\mathrm{sp}}(z, \tau) e^{-i\left(\omega-\omega_{0}\right) \tau} d \tau \\
& =\hbar \omega g(z, \omega) n_{\mathrm{sp}}(z, \omega)
\end{aligned}
$$

where $n_{\mathrm{sp}}$ is the population-inversion factor. When we are dealing with a narrow frequency range around the carrier frequency $\omega_{0}$, we can often assume that the spectrum is white, i.e., constant in frequency, and use the approximation $S_{\mathrm{sp}}(z, \omega) \approx S_{\mathrm{sp}}\left(z, \omega_{0}\right)$. The corresponding $R_{\mathrm{sp}}(z, \tau)$ is then

$$
R_{\mathrm{sp}}(z, \tau)=S_{\mathrm{sp}}\left(z, \omega_{0}\right) \delta(\tau) .
$$

However, a constant, unlimited noise spectrum means that the noise power is infinite, which leads to divergent terms in the second-order noise contributions. We shall therefore use the form

$$
R_{\mathrm{sp}}(z, \tau)=S_{\mathrm{sp}}\left(z, \omega_{0}\right) R(\tau)
$$

where $R(0)$ is finite and where the gain factor in $S_{\mathrm{sp}}\left(z, \omega_{0}\right)$ is the steady-state gain $g_{s}(z)$. For the population-inversion factor $n_{\mathrm{sp}}$, we use the approximation $n_{\mathrm{sp}} \approx\left(g_{s}+a N_{0}\right) / g_{s}$. In the simulations, we use the time step $\Delta t$ for the discrete sampling of the signal. By assuming that the signal is a sample-and-hold signal, i.e., the signal is constant between sampling points, the correlation in time becomes

$$
R(\tau)= \begin{cases}0, & |\tau| \geq \Delta t \\ \frac{1}{\Delta t}\left(1-\frac{|\tau|}{\Delta t}\right), & |\tau| \leq \Delta t .\end{cases}
$$


This correlation corresponds to a sinc ${ }^{2}$-shaped spectrum, which also limits the amount of noise power

$$
S_{\mathrm{sp}}(z, \omega)=S_{\mathrm{sp}}\left(z, \omega_{0}\right) \operatorname{sinc}^{2}\left(\left(\omega-\omega_{0}\right) \Delta t / 2\right) .
$$

Note that the unnormalized sinc function is used. As long as the sampling interval is sufficiently short to make the noise spectrum much wider than any filtering in the system, this assumption gives first-order results very similar to the white noise assumption.

The injected field is chosen to be of the form

$$
E(0, t)=E_{s}(0)(1+x(t)+i y(t))
$$

where $E_{s}$ is constant and the real functions $x(t)$ and $y(t)$ are Gaussian stochastic variables with zero mean and variance $\sigma^{2}$. They are sampled with time interval $\Delta t$ and satisfy the correlation relations

$$
\begin{aligned}
& \langle x(t) x(t+\tau)\rangle=\langle y(t) y(t+\tau)\rangle=\sigma^{2} R(\tau) \Delta t \\
& \langle x(t) y(t+\tau)\rangle=0
\end{aligned}
$$

with $R(\tau)$ given by (11). $\sigma$ is related to the input signal-to-noise ratio by noting that the input power has a noncentral $\chi^{2}$-distribution with mean and variance given by

$$
\begin{aligned}
\langle P\rangle & =P_{\text {in }}\left(1+2 \sigma^{2}\right) \\
\left\langle(\delta P)^{2}\right\rangle & =4 P_{\text {in }}^{2}\left(\sigma^{2}+\sigma^{4}\right)
\end{aligned}
$$

where $P_{\text {in }}=\left|E_{s}(0)\right|^{2}$. By comparing (13) with (1), we see that we must choose

$$
\begin{aligned}
& \frac{\rho_{1}(0, t)}{\rho_{s}(0)}=x(t), \quad \phi_{1}(0, t)=y(t) \\
& \frac{\rho_{2}(0, t)}{\rho_{s}(0)}=\frac{1}{2} y^{2}(t), \quad \phi_{2}(0, t)=-x(t) y(t)
\end{aligned}
$$

to ensure that (1) is satisfied to second order.

\section{A. Large-Signal Simulations}

In order to have a comparison for our second-order model, we have implemented a brute-force large-signal model, which uses statistical methods for simulating the PDF of the signal. The model has been presented in detail in [13] and is based on the work in [17]. The rate equations (4) and (5) are integrated numerically by discretizing the signal in time and the SOA in the $z$-direction. Noise terms with statistics according to (14) and (7) are added to the signal field at the input and for each SOA section, respectively. At the output of the device, the signal field is filtered using an optical filter and then detected assuming an ideal noiseless detector including a low-pass electrical filter with filter function $F_{e}$. The statistics of the detected signal are then extracted, and the PDF is estimated by making a histogram of the signal.

\section{B. Perturbation Expansion}

The perturbation analysis of (4) and (5) is based on the assumption that the Langevin noise term $f_{E}$ is small compared with the signal field and can be considered as a perturbation. The first step in the analysis is to derive the steady-state solution for $f_{E}=0$. As mentioned above, we will throughout the paper assume that $f_{N}=0$.

Equations (6) and (4) lead to the following equation for the gain:

$$
\frac{\partial g}{\partial t}=\frac{g_{0}-g}{\tau_{s}}-\frac{g|E|^{2}}{\tau_{s} P_{\text {sat }}}
$$

where $P_{\text {sat }}$ is the saturation power

$$
P_{\mathrm{sat}}=\frac{A \hbar \omega_{0}}{a \tau_{s}}
$$

and $g_{0}$ is the unsaturated gain, i.e., the steady-state gain when $|E|^{2}=0$ as

$$
g_{0}=a\left(\frac{I}{q V}+\frac{N_{0}}{\tau_{s}}\right) .
$$

The steady-state solution to (19) becomes

$$
g_{s}(z)=\frac{g_{0}}{1+\rho_{s}^{2}(z)}
$$

where $\rho_{s}$ is the steady-state normalized field amplitude introduced in (2). For $f_{E}=0$, integration of (5) gives

$$
E_{s}(z)=E_{s}(0) \exp \left(\frac{1}{2} \int_{0}^{z}\left((1-i \alpha) g_{s}\left(z^{\prime}\right)-\alpha_{i n t}\right) d z^{\prime}\right) \text {. }
$$

Equations (22) and (23) can be solved numerically for given input field $E_{s}(0)$.

In (1), the envelope field was factorized as $E=E_{s} E_{p}$, where the factor $E_{p}$ describes the perturbations due to noise as

$$
E_{p}=\left(1+\frac{\rho_{1}}{\rho_{s}}+\frac{\rho_{2}}{\rho_{s}}+\cdots\right) e^{i\left(\phi_{1}+\phi_{2}+\cdots\right)} .
$$

By inserting $E=E_{s} E_{p}$ into (5), it follows that $E_{p}$ satisfies the equation

$$
\frac{d E_{p}}{d z}=\frac{1}{2}\left(g-g_{s}\right)(1-i \alpha) E_{p}+\frac{f_{E}}{E_{s}} .
$$

Equations (19) and (25) can be solved by inserting the expansions (24) for $E_{p}$ and

$$
g=g_{s}+g_{1}+g_{2}+\cdots
$$

for the gain and equating terms of the same order in $f_{E} / E_{s}$. The first-order equations become

$$
\begin{aligned}
\frac{\partial}{\partial z}\left(\frac{\rho_{1}}{\rho_{s}}\right) & =\frac{1}{2} g_{1}+N_{\rho} \\
\frac{\partial}{\partial z} \phi_{1} & =-\frac{\alpha}{2} g_{1}+N_{\phi} \\
\tau_{s} \frac{\partial g_{1}}{\partial t} & =-g_{1}-\rho_{s}^{2} g_{1}-2 g_{s} \rho_{s} \rho_{1}
\end{aligned}
$$


where $N_{\rho}=\operatorname{Re}\left(f_{E} / E_{s}\right)$ and $N_{\phi}=\operatorname{Im}\left(f_{E} / E_{s}\right)$. The equations for the second-order terms become

$$
\begin{aligned}
\frac{\partial}{\partial z}\left(\frac{\rho_{2}}{\rho_{s}}\right)= & \frac{1}{2}\left(g_{2}+\frac{g_{1} \phi_{1}}{\rho_{s}}\right)+\phi_{1} N_{\phi} \\
\frac{\partial}{\partial z} \phi_{2}= & -\frac{\alpha}{2} g_{2}-\phi_{1} N_{\rho}-\frac{\rho_{1}}{\rho_{s}} N_{\phi} \\
\tau_{s} \frac{\partial g_{2}}{\partial t}= & -g_{2}-\rho_{s}^{2} g_{2} \\
& -\left(g_{s} \rho_{1}^{2}+2 \rho_{s} g_{1} \rho_{1}+2 g_{s} \rho_{s} \rho_{2}\right) .
\end{aligned}
$$

The frequency-domain solution of these equations are given in Appendix A.

Equations (27)-(32) also apply to the case where the incoming field is modulated, provided that $E_{s}=\sqrt{P_{\text {sat }}} \rho_{s} e^{\phi_{s}}$ and $g_{s}$ are the time-dependent field and gain solutions to (5) and (19) in the absence of noise $\left(f_{E}=0\right)$.

\section{PDF OF A FILTERED OUtPUT SignAL}

The output field from the SOA is assumed to be filtered by an optical filter with a bandwidth that is much smaller than the bandwidth of the spontaneous emission spectrum. The filter is described by a time response function $F(t)$, which we assume to be real to ensure that the filtering is symmetric around the carrier frequency $\omega_{0}$. The filtered output field is then $E_{\mathrm{F}}(t)=$ $(F * E)(L, t)$, where $*$ means convolution in the time domain. For the filtered output power, we obtain an expression similar to (3) as

$$
\begin{aligned}
P_{F}(t)=\left|E_{\mathrm{F}}\right|^{2} & \\
=P_{s}\left[\left(1+F * \frac{\rho_{1}}{\rho_{s}}\right)^{2}+2 F * \frac{\rho_{2}}{\rho_{s}}\right. & \\
& \left.\quad+\left(F * \phi_{1}\right)^{2}-F * \phi_{1}^{2}+\ldots\right]_{z=L} .
\end{aligned}
$$

We notice that the filtering induces a second-order phase-to-intensity conversion. The expression reduces to (3) when $F(t)=$ $\delta(t)$.

The aim of this section is to calculate the PDF of $P_{F}(t)$. Since the power is assumed to be stationary, it is sufficient to determine the PDF for $t=0$. The procedure is first to derive an expression for the moment generating function (MGF)

$$
\Phi_{P}(s)=\left\langle e^{-s P_{F}(0)}\right\rangle .
$$

The PDF of $P_{F}$ is then obtained from the inverse Laplace transform

$$
\operatorname{PDF}\left(P_{F}\right)=\frac{1}{2 \pi i} \int_{s_{0}-i \infty}^{s_{0}+i \infty} \Phi_{P}(s) \mathrm{e}^{s P_{F}} d s
$$

where $s_{0}$ is a real number for which $\Phi_{P}(s)$ has no singularities for $\operatorname{Re}(s) \geq s_{0}$. In order to describe the set of frequency-domain noise functions $\left\{x(\omega), y(\omega), N_{\rho}(z, \omega), N_{\phi}(z, \omega)\right\}$, it is convenient to introduce a vector space spanned by the vectors $|z, \omega, \alpha\rangle$ and $|\omega, \alpha\rangle$, where $z \in[0, L], \omega$ is real, and $\alpha$ is the label $\rho$ or $\phi$. The vectors are assumed to satisfy the orthogonality relations

$$
\begin{aligned}
\left\langle z_{1}, \omega_{1}, \alpha \mid z_{2}, \omega_{2}, \beta\right\rangle & =\delta\left(z_{1}-z_{2}\right) 2 \pi \delta\left(\omega_{1}-\omega_{2}\right) \delta_{\alpha \beta} \\
\left\langle\omega_{1}, \alpha \mid \omega_{2}, \beta\right\rangle & =2 \pi \delta\left(\omega_{1}-\omega_{2}\right) \delta_{\alpha \beta} \\
\left\langle z_{1}, \omega_{1}, \alpha \mid \omega_{2}, \beta\right\rangle & =0
\end{aligned}
$$

A set of noise functions is then described by a vector $|n\rangle$ with components

$$
\begin{aligned}
\langle z, \omega, \alpha \mid n\rangle & =N_{\alpha}(z, \omega) \\
\langle\omega, \rho \mid n\rangle & =x(\omega) \\
\langle\omega, \phi \mid n\rangle & =y(\omega)
\end{aligned}
$$

i.e., the vector $|n\rangle$ describes the real and imaginary parts of both the noise added along the length of the amplifier (39) and the noise of the input signal (40), which is independent of $z$. Using this notation, it is shown in Appendix A that the filtered output power can be written as

$$
\begin{aligned}
P_{F}(0) & =P_{s}\left[(1+\langle k \mid n\rangle)^{2}+\langle n|\mathcal{O}| n\rangle\right] \\
& =P_{s}[1+2\langle k \mid n\rangle+\langle n|\mathcal{Q}| n\rangle]
\end{aligned}
$$

where $|k\rangle$ is the vector with components (70) and $\mathcal{Q}$ is the operator

$$
\mathcal{Q}=|k\rangle\langle k|+\mathcal{O}
$$

$\mathcal{O}$ is the operator with matrix elements given by (71)-(73).

The probability of observing a particular noise vector $|n\rangle$ is assumed to be given by the Gaussian functional (see, e.g., [19, eq. (12.41)])

$$
\mathcal{P}[|n\rangle]=N \mathrm{e}^{-\frac{1}{2}\langle n|\mathcal{A}| n\rangle}
$$

where $N$ is a normalization constant that ensures that $\langle 1\rangle=$ 1. $\mathcal{A}$ is the reciprocal of the diffusion operator $\mathcal{D}$ with matrix elements given in Appendix B. For $P_{F}(0)$ given by (41) and for the Gaussian noise distribution (43), the MGF (34) is the path integral

$$
\begin{aligned}
\Phi_{P}(s)=N \int \exp [ & -s P_{s}(1+2\langle k \mid n\rangle+\langle n|\mathcal{Q}| n\rangle) \\
& \left.-\frac{1}{2}\langle n|\mathcal{A}| n\rangle\right] d[|n\rangle] .
\end{aligned}
$$


The integral can be shown to be given by the explicit form (see, e.g., [20, eq. (1.12)])

$$
\Phi_{P}(s)=\frac{\left.\exp \left[-s P_{s}+2\left(s P_{s}\right)^{2}\left\langle k\left|\left(\mathcal{A}+2 s P_{s} \mathcal{Q}\right)^{-1}\right| k\right\rangle\right)\right]}{\sqrt{\operatorname{det}\left(1+2 s P_{s} \mathcal{D Q}\right)}} .
$$

The MGF can be calculated directly by discretizing the space and frequency intervals.

From the MGF, it is straightforward to derive the mean value and the central moments (see, for example, [21]) by using the cumulant-generating function (CGF)

$$
\mathrm{CGF}(s)=\ln \left(\Phi_{P}(s)\right) .
$$

From the CGF, the cumulants of order $n$ can be calculated by

$$
k_{n}=\left.(-1)^{n} \frac{\partial^{n} \mathrm{CGF}(s)}{\partial s^{n}}\right|_{s=0} .
$$

The first-order cumulant is identical to the mean value, and orders two and three are identical to the respective central moments defined by

$$
m_{n}=\int_{-\infty}^{\infty}\left(P_{F}-\left\langle P_{F}\right\rangle\right)^{n} \operatorname{PDF}\left(P_{F}\right) d P_{F} .
$$

The distribution thus has the mean, variance, and third-order moments

$$
\begin{aligned}
\left\langle P_{F}\right\rangle & =P_{s}(1+\operatorname{Tr}[\mathcal{D} \mathcal{Q}]) \\
\left\langle\left(P_{F}-\left\langle P_{F}\right\rangle\right)^{2}\right\rangle & =2 P_{s}^{2}\left(\operatorname{Tr}\left[(\mathcal{D} \mathcal{Q})^{2}\right]+2\langle k|\mathcal{D}| k\rangle\right) \\
\left\langle\left(P_{F}-\left\langle P_{F}\right\rangle\right)^{3}\right\rangle & =8 P_{s}^{3}\left(\operatorname{Tr}\left[(\mathcal{D} \mathcal{Q})^{3}\right]+3\langle k|\mathcal{D} \mathcal{Q D}| k\rangle\right) .
\end{aligned}
$$

The first term in (50) corresponds to the spontaneous-spontaneous beat noise while the second term represents the signal-spontaneous beat noise.

It is shown in Appendix $C$ that (45) reduces to the familiar MGF for a noncentral $\chi^{2}$-distribution when the operator $\mathcal{O}$ in (41) is zero as

$$
\Phi_{P}(s)=\frac{1}{\sqrt{1+2 s P_{s}\langle k|\mathcal{D}| k\rangle}} \exp \left[\frac{-s P_{s}}{1+2 s P_{s}\langle k|\mathcal{D}| k\rangle}\right] .
$$

The distribution has the mean value and variance

$$
\begin{aligned}
\left\langle P_{F}\right\rangle & =P_{s}(1+\langle k|\mathcal{D}| k\rangle) \\
\left\langle\left(P_{F}-\left\langle P_{F}\right\rangle\right)^{2}\right\rangle & =2 P_{s}^{2}\left(|\langle k|\mathcal{D}| k\rangle|^{2}+2\langle k|\mathcal{D}| k\rangle\right) .
\end{aligned}
$$

By (70) and (77)-(79), the parameter $\langle k|\mathcal{D}| k\rangle$ can be written as

$$
\begin{aligned}
&\langle k|\mathcal{D}| k\rangle=\frac{1}{2 \pi} \int_{-\infty}^{\infty} d \omega {\left[| F ( \omega ) | ^ { 2 } \left(\int_{0}^{L}|H(z, \omega)|^{2} D_{\rho \rho}(z, \omega) d z\right.\right.} \\
&\left.\left.+|H(0, \omega)|^{2} D_{0}(\omega)\right)\right]
\end{aligned}
$$

The expression (55) (multiplied by four) can be shown to be the frequency integral over the RIN spectrum.

Thus far, we have treated the optical field and power, but, in order to compare with measurements, we need to introduce the conversion to the electrical domain by a detector. The detector model used here is an ideal noiseless square-law detector with unit responsivity and a limited frequency response. The effect on the PDF can thus be calculated by introducing a second filter acting on the optical power, which is the same as filtering the electrical current from the detector. The time-dependent current is then

$$
I(t)=F_{e}(t) * P_{F}(t)
$$

where $F_{e}(t)$ is the filter function of the detector. The result for the MGF expressed by (45) agrees with (56) if a new operator $\mathcal{R}$, with matrix elements given in Appendix A (74)-(76), and a new vector $|l\rangle$, with components as shown for $|k\rangle$ in (70) but multiplied by $F_{e}^{*}(\omega)$, are substituted for $\mathcal{Q}$ and $|k\rangle$, respectively, in (45). The operator $\mathcal{R}$ is very similar to $\mathcal{Q}$, but the electrical filtering means that the matrix elements are multiplied by $F_{e}\left(\omega_{2}-\omega_{1}\right)$.

In Section IV, we present a numerical analysis of the effect on the PDF of $\mathcal{O}$ and the electrical filter.

\section{NumERICAL EXAMPLES AND DISCUSSION}

Here, the model is used to analyze a few specific examples. The examples are chosen to be similar to the ones in [11] in order to be able to compare with the measurements in that work. The PDFs from the second-order model are calculated by discretizing space and frequency and numerically solving (45) and (35). The matrix calculations, and especially calculating the determinant, requires a large computer memory when a fine discretization is used. In order to reduce the requirements and allow better numerical resolution, we instead calculate an appropriate number of terms in the sums of the expansions in (94) and (95) [with $\mathcal{W}$ replaced by $\mathcal{U}$ in (94)], as described in Appendix C. For each higher order moment to be included in the investigation, another term has to be included in the sums when calculating the MGF. In this investigation, we have limited ourself to the third-order moment and, hence, three terms of (94) and (95).

The parameters used in the calculations are a mixture of known physical parameters for the measurements of [11] (i.e., length of SOA, bias current, input powers and detection bandwidth), reasonable guesses (e.g., coupling losses, waveguide losses, linewidth enhancement factor, and carrier lifetime) and fitted parameters (i.e., small-signal gain, saturation power, and input signal-to-noise ratio). The fitted parameter values are chosen to give a qualitatively reasonable fit to the experimental results in [11] for both the standard deviation and the skewness, which is defined as the normalized third-order central moment of the distributions. No quantitative fitting procedure has been carried out. The chosen parameter values are shown in Table I. The filters used in the calculations are eighth-order Butterworth filters.

First, we will compare the PDFs. Fig. 1 shows the PDFs calculated with $(\mathcal{O} \neq 0)$ and without $(\mathcal{O}=0)$ the proper inclusion of the second-order terms, as well as the simulations. The usefulness of analytical expressions for the PDFs is clearly seen when comparing the tails of the measured and simulated PDFs. 
TABLE I

Parameter VAlues USED in the CALCUlations

\begin{tabular}{|l|c|}
\hline Parameter & Value \\
\hline Device length, $L[\mu \mathrm{m}]$ & 500 \\
Bias current, $I[\mathrm{~mA}]$ & 200 \\
Input SNR at $10 \mathrm{GHz},[\mathrm{dB}]$ & 26 \\
Detection bandwidth, $B_{e}[\mathrm{GHz}]$ & 10 \\
Optical filter bandwidth, $B_{o}[\mathrm{GHz}]$ & 30 \\
Total simulation bandwidth, $1 / \Delta t[\mathrm{GHz}]$ & 125 \\
Coupling losses SOA, [dB] & 4 \\
Transmission loss SOA to detector, [dB] & 8.79 \\
Time constant, $\tau_{\mathrm{s}}[\mathrm{ps}]$ & 100 \\
Saturation power, $P_{\mathrm{sat}}[\mathrm{mW}]$ & 6 \\
Internal loss, $\alpha_{\text {int }}\left[\mathrm{m}^{-1}\right]$ & 500 \\
Linewidth enhancement factor, $\alpha$ & 5 \\
Small signal gain, $g_{0}\left[\mathrm{~m}^{-1}\right]$ & 15789 \\
Differential modal gain, $a\left[\mathrm{~m}^{2}\right]$ & $3.77 \cdot 10^{-20}$ \\
Carrier density at transparency, $N_{0}\left[\mathrm{~m}^{-3}\right]$ & $1 \cdot 10^{24}$ \\
\hline
\end{tabular}

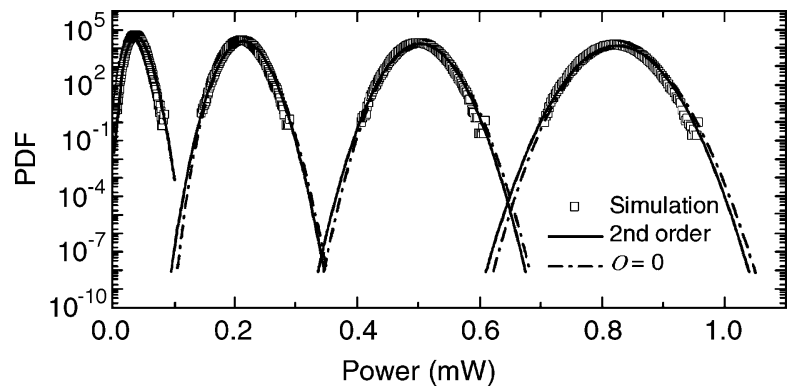

Fig. 1. Calculated PDFs at different power levels using the simulations and the small-signal model with (solid line) and without (dash-dotted line) proper inclusion of all second-order terms.

The simulations do not reach very far out in the tails and hence do not include low BERs. In order to extend the simulations further, within a reasonable simulation time, more advanced simulation techniques, for example, importance sampling [14], [15], has to be used or extrapolations have to be made [22]. The perturbation model is, however, able to reach far out in the tails using a reasonable computation time.

The difference between disregarding the second-order terms included in $\mathcal{O}$ and keeping them is not large when looking at the PDFs. However, if estimating the BER close to $10^{-10}$ the difference is more relevant, as seen in Fig. 2, where the difference gets close to one order of magnitude. This difference is also expected to be much larger for a more nonlinear system. The gain saturation model used in this work has proved to result in less nonlinear behavior than experiments [11]. It has also been shown that a more detailed model for the saturation of SOAs that takes into account counterpropagating ASE and includes nonzero facet reflectivities predicts a stronger nonlinearity [23]. In such a case of stronger nonlinearity, the role of higher order noise correlations is expected to be even more important.

The redistribution of the PDFs in the different models can be more quantitatively compared by considering the second- and third-order central moments or, equivalently, the standard deviation and skewness of the distributions, which are shown in Figs. 3 and 4. The results demonstrate the nonlinear noise redistribution due to the gain saturation in the SOA, as is discussed in more detail for the experiments in [11]. In this study, we will focus on the differences between the models. For the standard

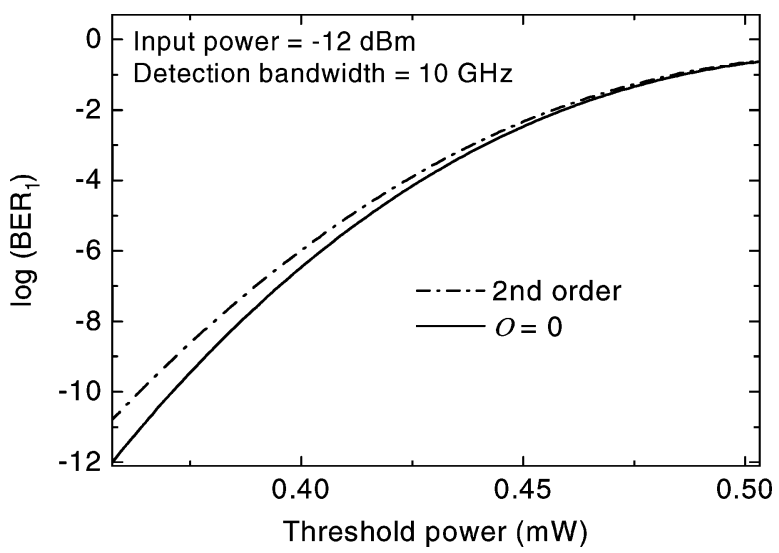

Fig. 2. BER of the $\mathrm{CW}$ signal corresponding to a long string of one bits as a function of decision threshold. The BER is calculated from the PDFs using the full second-order description (dash-dotted line) and when $\mathcal{O}=0$ (solid line) at input power $-12 \mathrm{dBm}$ and detection bandwidth $10 \mathrm{GHz}$.

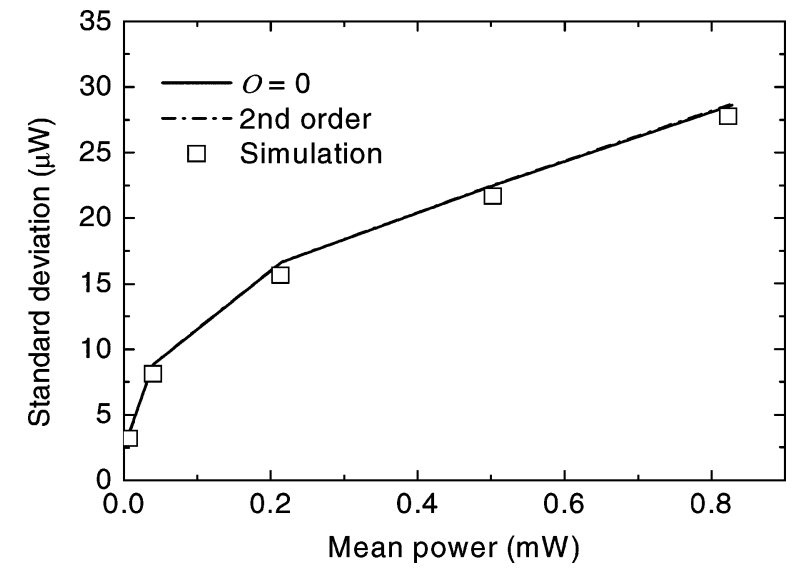

Fig. 3. Standard deviations of the calculated PDFs of a CW signal after the SOA as a function of mean optical power. Markers denote the simulation results, and the dash-dotted and solid lines (overlapping) denote the results of the full second-order description and when $\mathcal{O}=0$, respectively.

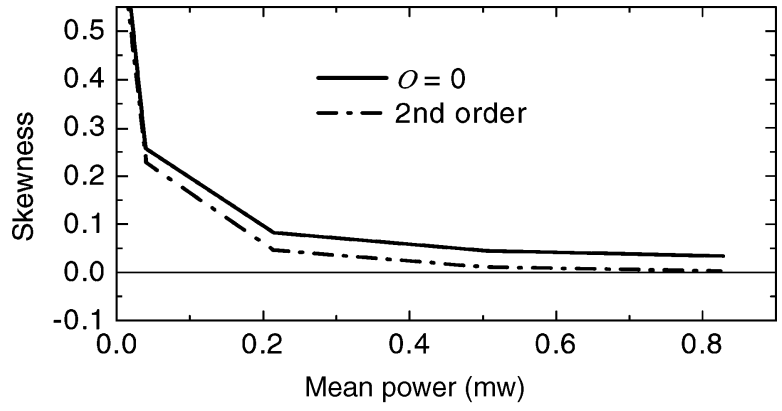

Fig. 4. Skewness of the calculated PDFs of a CW signal after the SOA as function of mean optical power. The dash-dotted and solid lines denote the results of the full second-order description and when $\mathcal{O}=0$, respectively.

deviation, they all give very similar results, as seen by the overlapping lines in Fig. 3.

In Fig. 4, the symmetry of the distributions are investigated by plotting the skewness, which is defined as

$$
\gamma_{s}=\frac{m_{3}}{m_{2}^{3 / 2}}
$$




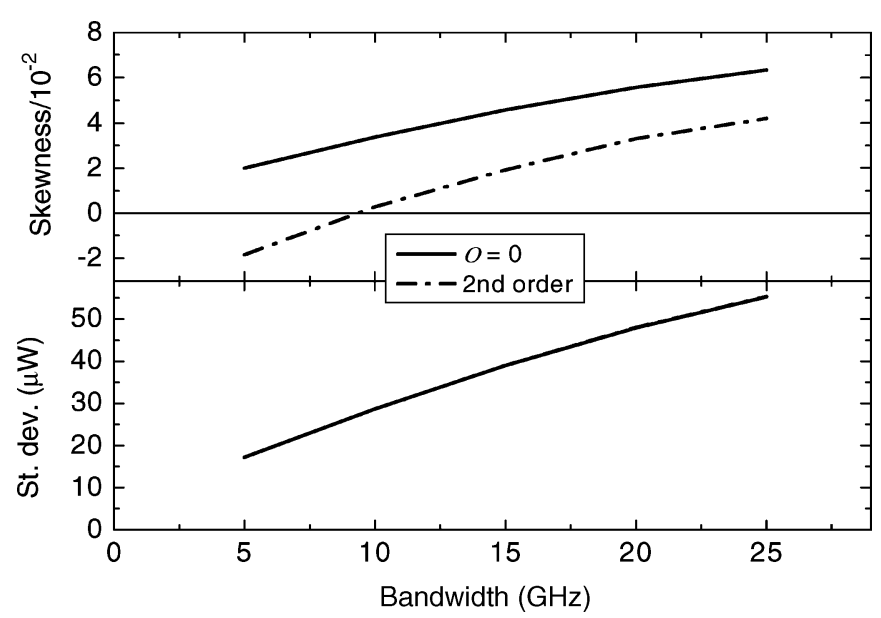

Fig. 5. Skewness (upper panel) and standard deviation (lower panel) of the calculated PDFs versus detection bandwidth for the full second-order model (dash-dotted line) and when the operator $\mathcal{O}$ is zero (solid line).

where $m_{i}$ is the $i$ th-order central moment. The skewness thus gives a measure of how much the noise distribution has been reshaped by the SOA nonlinearity, with a negative value of the skewness corresponding to a dominating low-power tail and a positive value indicating a dominating high-power tail of the distribution. It is worth noting that a noncentral $\chi^{2}$-distribution always has a positive skewness.

The solid and dash-dotted lines in Fig. 4 represent the secondorder model with and without the second-order terms described by the operator $\mathcal{O}$. The simulations do not give accurate results for the third-order moment due to a limited number of realizations (i.e., limited simulation time) and are therefore omitted in this figure. However, the qualitative results of the simulations do agree with the full second-order model. Fig. 4 shows that the nonlinear noise redistribution in the SOA skews the distribution in the negative direction compared to the noncentral $\chi^{2}$-distribution. This effect is seen in the simulations (not shown) and second-order model, just like in the experiments in [11], but only when all of the second-order terms are included $(\mathcal{O} \neq 0)$. This indicates that higher order models or statistical simulations are needed for properly describing the nonlinear noise redistribution in saturated SOAs.

The redistribution of noise in the SOA depends on the carrier dynamics in the amplifier, and, thus, the effect is only noticeable within the limited modulation bandwidth of the SOA. By varying the detection bandwidth in Fig. 5 the dependence on the dynamics can be mapped out. For a small detection bandwidth, most of the detected noise is inside the response bandwidth of the SOA and hence experiences a strong redistribution, resulting in a more negatively skewed distribution. The second-order model shows the possibility of achieving negative skewness, as seen in the experiments in [11], which the noncentral $\chi^{2}$-distribution cannot. For a larger detection bandwidth, however, a large part of the noise is not influenced by the SOA nonlinearity and the effect of including the full secondorder model is less pronounced, as seen in the upper panel of Fig. 5. In the lower panel of Fig. 5, the standard deviation is plotted as a function of detection bandwidth. As seen before, the full second-order model and the noncentral $\chi^{2}$-distribution give identical results, and the two lines overlap for the full range of investigated detection bandwidths.

\section{CONCLUSION}

We have developed a second-order perturbation model for describing the nonlinear redistribution of noise in saturated SOAs. A realistic example that was analyzed using the model shows that it can qualitatively describe the change in skewness of the distribution and calculate the PDFs far out in the tails, corresponding to very low BER. The full second-order model is needed for correctly describing the nonlinear redistribution of the PDFs by the saturated SOA, as described by the standard deviation and skewness, while the noncentral $\chi^{2}$-distribution only describes the change of the standard deviation. For the presented case of a fairly weak nonlinearity, the difference between the second-order model and the more standard noncentral $\chi^{2}$-distribution is small, but still leads to a difference in BER of about one order of magnitude at low BER. The difference is expected to be much more relevant if the model is applied to, for example, optical regenerators with strong nonlinearities in the decision gate.

Future studies may extend the formalism to modulated signals and include the effects of counterpropagating ASE and nonzero facet reflectivities. The case of low input powers, where the ASE cannot be treated as a perturbation of the signal, requires a modified approach, e.g., an iterative solution of (5).

\section{APPENDIX A}

This Appendix presents the derivation of the expressions for the vector $|k\rangle$ and the operator $\mathcal{O}$ in the expression (41) for the filtered output power $P_{F}(0)$.

It requires solution of (27)-(32) for the first- and second-order terms. Solving (29) and (32) in the frequency domain yields

$$
\begin{aligned}
& g_{1}(z, \omega)=2 C \frac{\rho_{1}}{\rho_{s}} \\
& g_{2}(z, \omega)=C\left[\frac{\rho_{1}}{\rho_{s}} \otimes \frac{\rho_{1}}{\rho_{s}}+2 \frac{g_{1}}{g_{s}} \otimes \frac{\rho_{1}}{\rho_{s}}+2 \frac{\rho_{2}}{\rho_{s}}\right]
\end{aligned}
$$

where $C$ is the function

$$
C(z, \omega)=-\frac{g_{s} \rho_{s}^{2}}{1+\rho_{s}^{2}+i \omega \tau_{s}}
$$

The symbol $\otimes$ means convolution in the frequency domain. For simplicity, we use the same notation for the frequency- and timedomain functions. The actual domain should be clear from context. The frequency-domain equations for the first- and secondorder amplitude and the first-order phase then become

$$
\begin{aligned}
\frac{\partial}{\partial z}\left(\frac{\rho_{1}}{\rho_{s}}\right) & =C \frac{\rho_{1}}{\rho_{s}}+N_{\rho} \\
\frac{\partial}{\partial z}\left(\phi_{1}\right) & =-\alpha C \frac{\rho_{1}}{\rho_{s}}+N_{\phi} \\
\frac{\partial}{\partial z}\left(\frac{\rho_{2}}{\rho_{s}}\right) & =C \frac{\rho_{2}}{\rho_{s}}+B
\end{aligned}
$$


where $B$ is the second-order driving term

$B(z, \omega)=\frac{g_{1}}{2} \otimes \frac{\rho_{1}}{\rho_{s}}+C\left[\frac{1}{2} \frac{\rho_{1}}{\rho_{s}} \otimes \frac{\rho_{1}}{\rho_{s}}+\frac{g_{1}}{g_{s}} \otimes \frac{\rho_{1}}{\rho_{s}}\right]+\phi_{1} \otimes N_{\phi}$.

The solutions to (61), (63), and (62) are

$$
\begin{aligned}
\frac{\rho_{1}(z, \omega)}{\rho_{s}(z)}= & \left(H(0, \omega) x(\omega)+\int_{0}^{z} H\left(z^{\prime}, \omega\right) N_{\rho}\left(z^{\prime}, \omega\right) d z^{\prime}\right) \\
& \times H(z, \omega)^{-1} \\
\frac{\rho_{2}(L, \omega)}{\rho_{s}(L)}= & \frac{1}{2} H(0, \omega)(y \otimes y)(\omega) \\
& +\int_{0}^{L} H\left(z^{\prime}, \omega\right) B\left(z^{\prime}, \omega\right) d z^{\prime} \\
\phi_{1}(z, \omega)= & y(\omega)-\alpha\left(\frac{H(0, \omega)}{H(z, \omega)}-1\right) x(\omega) \\
& +\int_{0}^{z}\left[\alpha\left(1-\frac{H\left(z^{\prime}, \omega\right)}{H(z, \omega)}\right) N_{\rho}\left(z^{\prime}, \omega\right)\right. \\
& \left.+N_{\phi}\left(z^{\prime}, \omega\right)\right] d z^{\prime}
\end{aligned}
$$

where

$$
H(z, \omega)=\exp \left(\int_{z}^{L} C\left(z^{\prime}, \omega\right) d z^{\prime}\right)
$$

and where we have inserted the initial values at $z=0$ from (17) and (18).

The filtered output power $P_{F}(0)$ is the frequency integral over the Fourier transform of $P_{F}(t)$ given in (33). It therefore follows that

$$
\begin{aligned}
P_{F}(0)=P_{s} & {\left[\left(1+\frac{1}{2 \pi} \int_{-\infty}^{\infty} F(\omega) \frac{\rho_{1}(L, \omega)}{\rho_{s}(L)} d \omega\right)^{2}\right.} \\
& +2 \frac{1}{2 \pi} \int_{-\infty}^{\infty} F(\omega) \frac{\rho_{2}(L, \omega)}{\rho_{s}(L)} d \omega \\
& +\frac{1}{(2 \pi)^{2}} \int_{-\infty}^{\infty}\left(F^{*}\left(\omega_{1}\right) F\left(\omega_{2}\right)-F\left(\omega_{2}-\omega_{1}\right)\right) \\
& \left.\times \phi_{1}^{*}\left(\omega_{1}\right) \phi_{1}\left(\omega_{2}\right) d \omega_{1} d \omega_{2}\right]
\end{aligned}
$$

The integral in the first squared parenthesis can be written as $\langle k \mid n\rangle$, where $|k\rangle$ is the vector with components

$$
\langle z, \omega, \rho \mid k\rangle=F^{*}(\omega) H^{*}(z, \omega)
$$

and where $\langle\omega, \rho \mid k\rangle=\langle 0, \omega, \rho \mid k\rangle$ and all other components are zero. The sum of the remaining two integrals between the square brackets can be written as $\langle n|\mathcal{O}| n\rangle$, where the operator $\mathcal{O}$ has the following matrix elements:

$$
\begin{aligned}
& \left\langle z_{1}, \omega_{1}, \rho|\mathcal{O}| z_{2}, \omega_{2}, \rho\right\rangle \\
& =F\left(\omega_{2}-\omega_{1}\right) H^{*}\left(z_{1}, \omega_{1}\right) H\left(z_{2}, \omega_{2}\right) \\
& \times \int_{0}^{L} \frac{H\left(z, \omega_{2}-\omega_{1}\right)}{H^{*}\left(z, \omega_{1}\right) H\left(z, \omega_{2}\right)} \\
& \times\left[C\left(-\omega_{1}\right)+C\left(\omega_{2}\right)+C\left(\omega_{2}-\omega_{1}\right)\right. \\
& \left.+\frac{2}{g_{s}(z)} C\left(\omega_{2}-\omega_{1}\right)\left(C\left(-\omega_{1}\right)+C\left(\omega_{2}\right)\right)\right] \\
& \times \theta\left(z-z_{1}\right) \theta\left(z-z_{2}\right) d z \\
& +\left(F^{*}\left(\omega_{1}\right) F\left(\omega_{2}\right)-F\left(\omega_{2}-\omega_{1}\right)\right) \\
& \times \alpha^{2}\left(1-H^{*}\left(z_{1}, \omega_{1}\right)\right)\left(1-H\left(z_{2}, \omega_{2}\right)\right) . \\
& \left\langle z_{1}, \omega_{1}, \phi|\mathcal{O}| z_{2}, \omega_{2}, \rho\right\rangle \\
& =\left\langle z_{2}, \omega_{2}, \rho|\mathcal{O}| z_{1}, \omega_{1}, \phi\right\rangle^{*} \\
& =F\left(\omega_{2}-\omega_{1}\right) \alpha\left(1-\frac{H\left(z_{2}, \omega_{2}\right)}{H\left(z_{1}, \omega_{2}\right)}\right) \theta\left(z_{1}-z_{2}\right) \\
& \times H\left(z_{1}, \omega_{2}-\omega_{1}\right)+\left(F^{*}\left(\omega_{1}\right) F\left(\omega_{2}\right)-F\left(\omega_{2}-\omega_{1}\right)\right) \\
& \times \alpha\left(1-H\left(z_{2}, \omega_{2}\right)\right) \text {. } \\
& \left\langle z_{1}, \omega_{1}, \phi|\mathcal{O}| z_{2}, \omega_{2}, \phi\right\rangle \\
& =F\left(\omega_{2}-\omega_{1}\right)\left(\theta\left(z_{2}-z_{1}\right) H\left(z_{2}, \omega_{2}-\omega_{1}\right)\right. \\
& \left.+\theta\left(z_{1}-z_{2}\right) H\left(z_{1}, \omega_{2}-\omega_{1}\right)\right) \\
& +F^{*}\left(\omega_{1}\right) F\left(\omega_{2}\right)-F\left(\omega_{2}-\omega_{1}\right) \text {. }
\end{aligned}
$$

Here, $\theta$ is the Heaviside step function. The remaining matrix elements of $\mathcal{O}$ involving the vectors $|\omega, \rho\rangle$ and $|\omega, \phi\rangle$ are obtained by replacing $|0, \omega, \rho\rangle$ by $|\omega, \rho\rangle$ and $|0, \omega, \phi\rangle$ by $|\omega, \phi\rangle$ in (71)-(73). For $z_{1}=z_{2}=0$, we use the definition $\theta(0) \equiv 1 / 2$.

When the electrical filter of the detector is included the operator, $\mathcal{R}$ should be used instead of $\mathcal{Q}$. It has the following matrix elements:

$$
\begin{aligned}
& \left\langle z_{1}, \omega_{1}, \rho|\mathcal{R}| z_{2}, \omega_{2}, \rho\right\rangle \\
& \quad=F_{e}\left(\omega_{2}-\omega_{1}\right) F^{*}\left(\omega_{1}\right) H^{*}\left(z_{1}, \omega_{1}\right) F\left(\omega_{2}\right) H\left(z_{2}, \omega_{2}\right) \\
& \quad+F_{e}\left(\omega_{2}-\omega_{1}\right)\left\langle z_{1}, \omega_{1}, \rho|\mathcal{O}| z_{2}, \omega_{2}, \rho\right\rangle \\
& \left\langle z_{1}, \omega_{1}, \phi|\mathcal{R}| z_{2}, \omega_{2}, \rho\right\rangle \\
& \quad=F_{e}\left(\omega_{2}-\omega_{1}\right)\left\langle z_{1}, \omega_{1}, \phi|\mathcal{O}| z_{2}, \omega_{2}, \rho\right\rangle \\
& \left\langle z_{1}, \omega_{1}, \phi|\mathcal{R}| z_{2}, \omega_{2}, \phi\right\rangle \\
& \quad=F_{e}\left(\omega_{2}-\omega_{1}\right)\left\langle z_{1}, \omega_{1}, \phi|\mathcal{O}| z_{2}, \omega_{2}, \phi\right\rangle
\end{aligned}
$$

and so on in the same way as for $\mathcal{O}$.

\section{APPENDIX B}

The diffusion operator $\mathcal{D}$ has matrix elements that are given by the correlation relation between the noise functions

$$
\begin{aligned}
\left\langle z_{1}, \omega_{1}, \alpha|\mathcal{D}| z_{2}, \omega_{2}, \beta\right\rangle= & \left\langle N_{\alpha}^{*}\left(z_{1}, \omega_{1}\right) N_{\beta}\left(z_{2}, \omega_{2}\right)\right\rangle \\
= & D_{\alpha \beta}\left(z_{1}, \omega_{1}\right) \delta\left(z_{1}-z_{2}\right) \\
& \times 2 \pi \delta\left(\omega_{1}-\omega_{2}\right) \\
\left\langle\omega_{1}, \rho|\mathcal{D}| \omega_{2}, \rho\right\rangle= & \left\langle x^{*}\left(\omega_{1}\right) x\left(\omega_{2}\right)\right\rangle \\
= & D_{0}\left(\omega_{1}\right) 2 \pi \delta\left(\omega_{1}-\omega_{2}\right)
\end{aligned}
$$




$$
\begin{aligned}
\left\langle\omega_{1}, \phi|\mathcal{D}| \omega_{2}, \phi\right\rangle & =\left\langle y^{*}\left(\omega_{1}\right) y\left(\omega_{2}\right)\right\rangle \\
& =D_{0}\left(\omega_{1}\right) 2 \pi \delta\left(\omega_{1}-\omega_{2}\right) .
\end{aligned}
$$

All other matrix elements of $\mathcal{D}$ are zero. It follows from (7) and the definition of $N_{\rho}$ and $N_{\phi}$ that

$$
\begin{aligned}
D_{\rho \rho}(z, \omega) & =D_{\phi \phi}(z, \omega) \\
& =\frac{1}{4}\left(S_{\mathrm{sp}}\left(z, \omega_{0}+\omega\right)+S_{\mathrm{sp}}\left(z, \omega_{0}-\omega\right)\right) /\left|E_{s}(z)\right|^{2} \\
D_{\rho \phi}(z, \omega) & =D_{\phi \rho}^{*}(z, \omega) \\
& =\frac{1}{4 i}\left(S_{\mathrm{sp}}\left(z, \omega_{0}+\omega\right)-S_{\mathrm{sp}}\left(z, \omega_{0}-\omega\right)\right) /\left|E_{s}(z)\right|^{2} .
\end{aligned}
$$

If we assume the symmetric form (12) for the spontaneous emission spectrum, the expressions (80) and (81) reduce to

$$
D_{\rho \rho}(z, \omega)=D_{\phi \phi}(z, \omega)=\frac{S_{\mathrm{sp}}\left(z, \omega_{0}\right) \operatorname{sinc}^{2}(\omega \Delta t / 2)}{2|E s(z)|^{2}}
$$

and $D_{\rho \phi}=D_{\phi \rho}=0$. By (14), the diffusion function $D_{0}(\omega)$ becomes

$$
D_{0}=\sigma^{2} \operatorname{sinc}^{2}(\omega \Delta t / 2) \Delta t
$$

\section{APPENDIX C}

A numerical calculation of the MGF expression (45) involves a calculation of $\langle k \mid \psi\rangle$, where $|\psi\rangle$ is the vector

$$
|\psi\rangle=\left(\mathcal{A}+2 s P_{s} \mathcal{Q}\right)^{-1}|k\rangle .
$$

$\mathcal{D}$ is the reciprocal of $\mathcal{A}$, and (84) can therefore be written as

$$
\left(1+2 s P_{s} \mathcal{D} \mathcal{Q}\right)|\psi\rangle=\mathcal{D}|k\rangle .
$$

This is a Fredholm integral equation of the second kind [24] from which $|\psi\rangle$ can be calculated by conventional integral equation methods. If we ignore the operator $\mathcal{O}$ in (42), the operator $1+2 s P_{s} \mathcal{D} \mathcal{Q}$ reduces to

$$
\mathcal{M} \equiv 1+2 s P_{s} \mathcal{D}|k\rangle\langle k| .
$$

We can therefore study the influence of the operator $\mathcal{O}$ by introducing the factorization

$$
1+2 s P_{s} \mathcal{D} \mathcal{Q}=\mathcal{M}(1+\mathcal{W})
$$

where

$$
\mathcal{W}=\mathcal{M}^{-1} 2 s P_{s} \mathcal{D O} .
$$

By using the explicit expression

$$
\mathcal{M}^{-1}=1-\frac{2 s P_{s} \mathcal{D}|k\rangle\langle k|}{1+2 s P_{s}\langle k|\mathcal{D}| k\rangle}
$$

(85) can be written as

$$
(1+\mathcal{W})|\psi\rangle=\frac{\mathcal{D}|k\rangle}{1+2 s P_{s}\langle k|\mathcal{D}| k\rangle}
$$

which is again a Fredholm integral equation. The formal solution for $\langle k \mid \psi\rangle$ is

$$
\langle k \mid \psi\rangle=\frac{1}{1+2 s P_{s}\langle k|\mathcal{D}| k\rangle} \sum_{\nu=0}^{\infty}\left\langle k\left|(-\mathcal{W})^{\nu} \mathcal{D}\right| k\right\rangle
$$

which may be used when the sum is rapidly converging.

To calculate the MGF from (45), we also need to determine the determinant

$$
\operatorname{det}\left(1+2 s P_{s} \mathcal{D} \mathcal{Q}\right)=\operatorname{det}(\mathcal{M}) \operatorname{det}(1+\mathcal{W}) .
$$

Since $\mathcal{D}^{1 / 2}|k\rangle$ is an eigenvector of $\mathcal{D}^{-1 / 2} \mathcal{M D}^{1 / 2}$ with eigenvalue $1+2 s P_{s}\langle k|\mathcal{D}| k\rangle$ and all vectors orthogonal to $\mathcal{D}^{1 / 2}|k\rangle$ are eigenvectors of $\mathcal{D}^{-1 / 2} \mathcal{M} \mathcal{D}^{1 / 2}$ with eigenvalue 1 , it follows that

$$
\operatorname{det}(\mathcal{M})=\operatorname{det}\left(\mathcal{D}^{-1 / 2} \mathcal{M D}^{1 / 2}\right)=1+2 s P_{s}\langle k|\mathcal{D}| k\rangle .
$$

The second factor in (92) may be calculated by using the expansion

$$
\begin{aligned}
\ln [\operatorname{det}(1+\mathcal{W})] & =\operatorname{Tr}[\ln (1+\mathcal{W})] \\
& =\sum_{\nu=1}^{\infty} \frac{(-1)^{\nu+1}}{\nu} \operatorname{Tr}\left(\mathcal{W}^{\nu}\right)
\end{aligned}
$$

where the symbol Tr means trace of the operator.

For $\mathcal{W}=0$, the MGF in (45) reduces to (52), which is the MGF of a noncentral $\chi^{2}$-distribution.

When the electrical filter of the detector is included, the factorization using $\mathcal{M}$ and $\mathcal{W}$ cannot be done. Instead, $|l\rangle$ and $\mathcal{R}$ have to be substituted for $|k\rangle$ and $\mathcal{Q}$, respectively, in the Fredholm integral equation described in (84) and (85). The solution is then

$$
\langle l \mid \psi\rangle=\sum_{\nu=0}^{\infty}\left\langle l\left|(-\mathcal{U})^{\nu} \mathcal{D}\right| l\right\rangle
$$

where $\mathcal{U}=2 s P_{s} \mathcal{D} \mathcal{R}$.

\section{REFERENCES}

[1] T. Durhuus, B. Mikkelsen, C. Jørgensen, S. L. Danielsen, and K. E. Stubkjær, "All-optical wavelength conversion by semiconductor optical amplifiers," J. Lightw. Technol., vol. 14, no. 6, pp. 942-954, Jun. 1996.

[2] B. Lee, R. Mohamad, and K. Dimyati, "Performance of all-optical multicasting via dual-stage XGM in SOA for grid networking," IEEE Photon. Technol. Lett., vol. 18, no. 12, pp. 2215-2217, Dec. 2006.

[3] O. Leclerc, B. Lavigne, E. Balmefrezol, P. Brindel, L. Pierre, D. Rouvillain, and F. Seguineau, "Optical regeneration at $40 \mathrm{~Gb} / \mathrm{s}$ and beyond," J. Lightw. Technol., vol. 21, no. 11, pp. 2779-2790, Nov. 2003.

[4] C. Bornholdt, J. Slovak, and B. Sartorius, "Semiconductor-based alloptical 3R regenerator demonstrated at $40 \mathrm{Gbit} / \mathrm{s}$," Electron. Lett., vol. 40, no. 3, pp. 192-193, 2004.

[5] Y. Su, X. Liu, and J. Leuthold, "Wide dynamic range 10-Gb/s DPSK packet receiver using optical-limiting amplifiers," IEEE Photon. Technol. Lett., vol. 16, no. 1, pp. 296-298, Jan. 2004.

[6] A. Mccoy, P. Horak, M. Ibsen, and D. Richardson, "Performance comparison of spectrum-slicing techniques employing SOA-based noise suppression at the transmitter or receiver," IEEE Photon. Technol. Lett., vol. 18 , no. 8, pp. 1494-1496, Aug. 2006. 
[7] M. Shtaif, B. Tromborg, and G. Eisenstein, "Noise spectra of semiconductor optical amplifiers: relation between semiclassical and quantum descriptions," IEEE J. Quantum Electron., vol. 34, no. 5, pp. 869-878, May 1998.

[8] M. Shtaif and G. Eisenstein, "Experimental study of the statistical properties of nonlinearly amplified signals in semiconductor optical amplifiers," IEEE Photon. Technol. Lett., vol. 9, no. 7, pp. 904-906, Jul. 1997.

[9] A. Bilenca and G. Eisenstein, "Statistical noise properties of an optical pulse propagating in a nonlinear semiconductor optical amplifier," IEEE J. Quantum Electron., vol. 41, no. 1, pp. 36-44, Jan. 2005.

[10] A. Bilenca and G. Eisenstein, "Fokker-Planck and Langevin analyses of noise accompanying the amplification of optical pulses in semiconductor optical amplifiers," J. Opt. Soc. Amer. B, Opt. Phys., vol. 22, no. 8, pp. 1632-1639, 2005.

[11] F. Öhman, B. Tromborg, J. Mørk, A. Aurelius, A. Djupsjöbacka, and A. Berntson, "Measurements and simulations of non-linear noise re-distribution in an SOA," IEEE Photon. Technol. Lett., vol. 17, no. 1, pp. 16-18, Jan. 2005.

[12] G. H. Einarsson, Principles of Lightwave Communication, 1st ed. New York: Wiley, 1996.

[13] F. Öhman, S. Bischoff, B. Tromborg, and J. Mørk, "Noise and regeneration in semiconductor waveguides with saturable gain and absorption," IEEE J. Quantum Electron., vol. 40, no. 3, pp. 245-255, Mar. 2004.

[14] R. Srinivasan, Importance Sampling-Applications in Communications and Detection. Berlin, Germany: Springer-Verlag, 2002.

[15] J. A. Bucklew, Introduction to Rare Event Simulation. New York: Springer-Verlag, 2004.

[16] G. Agrawal and N. Olsson, "Self-phase modulation and spectral broadening of optical pulses in semiconductor laser amplifiers," IEEE $J$. Quantum Electron., vol. 25, no. 11, pp. 2297-2306, Nov. 1989.

[17] D. Cassioli, S. Scotti, and A. Mecozzi, "A time-domain computer simulator of the nonlinear response of semiconductor optical amplifiers," IEEE J. Quantum Electron., vol. 36, no. 9, pp. 1072-1080, Sep. 2000.

[18] B. Tromborg, M. Shtaif, and G. Eisenstein, "Quantum description of noise in saturated semiconductor optical amplifiers: Analysis of generation of squeezed states," in Proc. 16th IEEE Int. Semicond. Laser Conf., 1998, pp. 35-36.

[19] R. Feynman and A. Hibbs, Quantum Mechanics and Path Integrals, 1st ed. New York: McGraw-Hill, 1965.

[20] J. Zinn-Justin, Path Integrals in Quantum Mechanics, 1st ed. Oxford, U.K.: Oxford Univ. Press, 2005.
[21] B. Saleh, Photoelectron Statistics. New York: Springer-Verlag, 1978.

[22] M. C. Jeruchim, P. Balaban, and K. S. Shanmugan, Simulation of Communication Systems, Modeling, Methodology and Techniques, 2nd ed. New York: Kluwer/Plenum, 2000.

[23] F. Ohman, R. Kjaer, L. Christiansen, K. Yvind, and J. Mork, "Steep and adjustable transfer functions of monolithic SOA-EA 2R regenerators," IEEE Photon. Technol. Lett., vol. 18, no. 9, pp. 1067-1069, Sep. 2006.

[24] J. Kondo, Integral Equations, 1st ed. Oxford, U.K.: Clarendon, 1981.

Filip Öhman received the M.Sc. degree in engineering from the Royal Institute of Technology (KTH), Stockholm, Sweden, in 2001, and the Ph.D. degree from the Technical University of Denmark, Lyngby, Denmark, in 2005.

He is currently with COM•DTU Institute for Communication Optics and Materials, Technical University of Denmark, Lyngby, where he is researching optical signal processing and noise in nonlinear semiconductor devices. In particular, he is investigating signal regeneration and applications in microwave photonics using semiconductor optical amplifiers and electro-absorbers.

Jesper Mørk received the Ph.D. and Dr. Techn. degrees from the Technical University of Denmark (DTU), Lyngby, in 1988 and 2003, respectively.

Since 2002, he has been a Professor with COM•DTU Institute for Communication, Optics and Materials, DTU. His current research interests are in the area of device physics, in particular, ultrafast devices for optical signal processing, noise in nonlinear devices, and quantum photonics.

Bjarne Tromborg was born 1940 in Give, Denmark. He received the M.Sc. degree in physics and mathematics from the Niels Bohr Institute, Copenhagen, Denmark, in 1968.

He continued as a University Researcher in high-energy particle physics. From 1979 to 1998, he was with the Danish Teleadministrations, where he was Head of Optical Communications Department, Tele Danmark Research, Horsholm (1987-1995). He was with COM•DTU Institute for Communication, Optics and Materials, Technical University of Denmark, Lyngby, from 1999 till he retired in June 2006, most of the time as a Research Professor. He has coauthored a research monograph and more than 100 journal and conference papers, mostly on the physics and technology of optoelectronic devises. 